\title{
A convolution thresholding scheme for the Willmore flow
}

\author{
RICHARDS GRZHIBOVSKIS ${ }^{\dagger}$ \\ Department of Mathematics, University of Saarland, D-66123 Saarbrücken, Germany \\ AleXeI HeINTZ \\ Department of Mathematics, Chalmers University of Technology, 41296 Göteborg, Sweden
}

[Received 8 December 2005 and in revised form 7 August 2007]

\begin{abstract}
A convolution thresholding approximation scheme for the Willmore geometric flow is constructed. It is based on an asymptotic expansion of the convolution of an indicator function with a smooth, isotropic kernel. The consistency of the method is justified when the evolving surface is smooth and embedded. Some aspects of the numerical implementation of the scheme are discussed and several numerical results are presented. Numerical experiments show that the method performs well even in the case of a non-smooth initial data.
\end{abstract}

Keywords: Willmore flow, convolution thresholding scheme.

\section{Introduction}

Let $\Sigma$ be a smooth compact and orientable surface. The Willmore functional is defined as

$$
W(\mathbf{f})=\int_{\Sigma} H^{2} \mathrm{~d} S
$$

for any immersion $\mathbf{f}: \Sigma \rightarrow \mathbb{R}^{3}$. Here $H$ denotes the mean curvature of $\Sigma$ and $\mathrm{d} S$ is the area measure. We refer to [26] for the general discussion of this functional as well as description of some stationary points. The variation of this integral for a perturbation $\phi$ of the surface along the normal is (see [26])

$$
\delta \mathcal{W}=\int_{\Sigma} \phi\left(\Delta H+2 H\left(H^{2}-K\right)\right) \mathrm{d} S,
$$

where $K$ is the Gaussian curvature, and $\Delta$ is the Laplace-Beltrami operator on $\Sigma$. The Willmore flow is an evolution of the surface where each point moves with the local normal velocity $v=$ $-\Delta H-2 H\left(H^{2}-K\right)$. More precisely, suppose $\mathbf{f}_{0}: \Sigma \rightarrow \mathbb{R}^{3}$ is a smooth immersion of a closed twodimensional manifold. The smooth Willmore flow with initial data $\mathbf{f}_{0}$ is a family $\mathbf{f}:[0, T] \times \Sigma \rightarrow \mathbb{R}^{3}$ of immersions $0<T \leqslant \infty$ such that $\mathbf{f}(0, \mathbf{x})=\mathbf{f}_{0}(\mathbf{x})$ and

$$
\frac{\partial \mathbf{f}(t, \mathbf{x})}{\partial t}=-\mathbf{N}(t, \mathbf{x})\left(\Delta H(t, \mathbf{x})+2 H(t, \mathbf{x})\left(H^{2}(t, \mathbf{x})-K(t, \mathbf{x})\right)\right)
$$

for $t \in[0, T]$. Here $\mathbf{N}(t, \mathbf{x})$ denotes the normal vector to $\mathbf{f}(t, \mathbf{x})$.

\footnotetext{
†E-mail: richards@num.uni-sb.de

‡E-mail: heintz@math.chalmers.se
} 
As an evolution that minimizes mean curvature this flow can be used for surface smoothing. We refer to [4] where the authors propose a numerical scheme for so called surface diffusion flow, i.e. the evolution of the surface with the normal velocity $v=-\Delta H$. An extension of this result and applications to the surface smoothing can be found in [25]. A numerical algorithm for surface restoration based on a finite element approximation for the Willmore flow was proposed in [5]. Another recent result devoted to the level-set formulation of the Willmore flow and the finite element approach to the evolution can be found in [7].

Another application of the Willmore flow comes from the biophysics. The functional (1) is a particular case of the Helfrich functional that describes the free energy of a bilayer membrane [3, 6]. A phase field approach to the problem is suggested in [8] and [9].

The mathematical properties of this type of surface evolution attracted much attention during the last years. The Willmore flow close to a sphere was studied in [24]. A numerical scheme for axisymmetric Willmore flows in $\mathbb{R}^{3}$ was proposed in [18]. A numerical example of occurrence of a self-intersection during the evolution is constructed in [19]. The case with small initial energy was considered by Kuwert and Schätzle in [14]. In [15] they have obtained a lower bound on the existence time of the smooth Willmore flow. This bound is expressed in terms of the initial concentration of the mean curvature. In [16] the existence result was developed further by the same authors. They have proved that the Willmore flow of a smooth embedding of a sphere into $\mathbb{R}^{3}$ exists, is smooth and converges to a round sphere provided that the initial energy is bounded by $8 \pi$. An attempt to define a kind of weak solution and to show its existence past singularities can be found in [21].

The purpose of the present paper is to develop a simple convolution thresholding scheme for tracking such evolutions of two-dimensional surfaces in $\mathbb{R}^{3}$. Convolution thresholding schemes have proved to be a useful tool for the numerics of the surface evolution [1, 20, 22, 23, 12, 13, 11]. Furthermore, the convolution structure of the method allows for a numerically efficient implementation.

This paper is organized as follows. In Section 2 we consider the graph of a smooth function and give elementary expressions for its geometrical characteristics (i.e. $H, K$ and $\Delta H$ ) in terms of the derivatives of the function. These expressions are used in Section 3, where we derive the asymptotic expansion of the convolution of the indicator function of a domain $C$ in $\mathbb{R}^{3}$ with an isotropic and fast decreasing kernel $\rho\left(\left|\mathbf{r}-\mathbf{r}_{0}\right|^{2} / t^{2 \alpha}\right) /\left(t^{\alpha}\right)^{3}$ when $t \rightarrow 0$ for points $\mathbf{r}_{0}$ close to $\partial C$. The key result is that the third term in the asymptotic expansion is proportional to $\Delta H+2 H\left(H^{2}-K\right)$ on $\partial C$. A linear combination of two such convolutions is used to construct a convolution thresholding scheme for the Willmore flow. The convergence of the scheme for smooth embedded solutions is established in Section 3 . The formulation of the method, however, is independent of the smoothness of $\Sigma$. Several numerical examples of the flow together with some implementation aspects are presented in Section 4 . Numerical experiments show that the method gives precise results even in cases with non-smooth initial data.

\section{Geometric properties of a smooth graph}

Let us consider the graph of a smooth function $f: \mathbb{R}^{2} \rightarrow \mathbb{R}$ such that $\partial f / \partial u_{i}(0,0)=f_{i}=0$ for $i=1,2$. We express the mean curvature $H$, the Gaussian curvature $K$, the Laplace-Beltrami operator of the mean curvature $\Delta H$ of the graph at the origin in terms of $f$ and its derivatives (see [26] for details). 
Consider the mapping $\mathbf{x}: \mathbb{R}^{2} \rightarrow \mathbb{R}^{3}$ given by $\mathbf{x}\left(u_{1}, u_{2}\right)=\left(u_{1}, u_{2}, f\left(u_{1}, u_{2}\right)\right)$. Set $\mathbf{x}_{i}=\partial \mathbf{x} / \partial u_{i}$ for $i=1,2$. The unit normal $\mathbf{N}$ of the graph is given by

$$
\mathbf{N}=\frac{\mathbf{x}_{1} \times \mathbf{x}_{2}}{\left|\mathbf{x}_{1} \times \mathbf{x}_{2}\right|} .
$$

The coefficients of the first fundamental form are $g_{i j}=\left\langle\mathbf{x}_{i}, \mathbf{x}_{j}\right\rangle$ and the second fundamental form has coefficients $h_{i j}=-\left\langle\mathbf{N}_{i}, \mathbf{x}_{j}\right\rangle$, where $\mathbf{N}_{i}=\partial \mathbf{N} / \partial u_{i}$. The mean curvature is

$$
H=\frac{1}{2} \sum_{i, j} g^{i j} h_{i j},
$$

where $g^{i j}$ denotes the elements of the matrix inverse to $g$. Setting $u_{1}=u_{2}=0$ we get

$$
H=\frac{1}{2}\left(f_{11}(0,0)+f_{22}(0,0)\right) .
$$

In order to calculate $\Delta H$ we use the Christoffel symbols

$$
\Gamma_{i j}^{k}=\frac{1}{2} \sum_{h} g^{h k}\left(\partial g_{i h} / \partial u_{j}+\partial g_{j h} / \partial u_{i}-\partial g_{i j} / \partial u_{h}\right) .
$$

The covariant derivatives of $H$ are

$$
\begin{aligned}
\nabla_{i} H & =H_{i}=\frac{\partial H}{\partial u_{i}}, \\
\nabla_{i} \nabla_{j} H & =H_{i j}-\sum_{k} \Gamma_{j i}^{k} H_{k} .
\end{aligned}
$$

The Laplacian of $H$ can be written as

$$
\Delta H=\sum_{i, j} g^{i j} \nabla_{i} \nabla_{j} H .
$$

After substituting (2)-(5) into (6) we get the following equality at the origin:

$$
\Delta H=\frac{1}{2}\left(\Delta(\Delta f)-2 H\left(12 H^{2}-8 K\right)\right),
$$

where $K$ is the Gaussian curvature,

$$
K=\frac{\operatorname{det}\left(h_{i j}\right)}{\operatorname{det}\left(g_{i j}\right)}=f_{11}(0,0) f_{22}(0,0)-f_{12}^{2}(0,0) .
$$

\section{Asymptotics of the convolution}

In this section we consider a bounded domain $C$ in $\mathbb{R}^{3}$ with a smooth boundary $\partial C$. We study the connection between local geometric properties of $\partial C$ and analytic properties of the convolution

$$
M(\mathbf{r})=\left(\chi_{C} \star \rho_{t^{1 / 4}}\right)(\mathbf{r}), \quad \mathbf{r} \in \mathbb{R}^{3},
$$


when $t \rightarrow 0$ at points $\mathbf{r}$ with distance of order $O(t)$ from $\partial C$. Here $\chi_{C}$ is the characteristic function of $C, \rho_{t^{\alpha}}(\mathbf{r})=\rho\left(|\mathbf{r}|^{2} / t^{2 \alpha}\right) /\left(t^{\alpha}\right)^{3}$. The function $\rho:(0, \infty) \rightarrow[0, \infty)$ is smooth, has compact support (or is exponentially decreasing) and is normalized by $\int_{\mathbb{R}^{3}} \rho_{1}(\mathbf{r}) \mathrm{d} \mathbf{r}=1$.

We choose an arbitrary point $\mathbf{p} \in \partial C$. $\mathbf{N}$ denotes the internal (with respect to $C$ ) unit normal to $\partial C$ in $\mathbf{p}$. The velocity of a geometric flow in the normal direction is denoted by $v \in \mathbb{R}$. After a short time period $t$ the point $\mathbf{p}$ moving with normal velocity $v$ would reach the point $\mathbf{r}_{0}+O\left(t^{2}\right)$, where $\mathbf{r}_{0}=\mathbf{p}+\mathbf{N} v t$. We will consider the asymptotics of $M(\mathbf{r})$ for $t \rightarrow 0$ at the point $\mathbf{r}_{0}$. The scaling $t^{1 / 4}$ in the kernel $\rho_{t^{1 / 4}}$ is chosen to obtain the term including velocity $v$ of the same order $t^{3 / 4}$ as the term with the Willmore operator $\left(\Delta H+2 H\left(H^{2}-K\right)\right)$ in the expansion of $M\left(\mathbf{r}_{0}\right)$ for $t \rightarrow 0$. By choosing the classical scaling $t^{1 / 2}$ one gets the expansion of $M$ where the velocity $v$ and the mean curvature $H$ appear both in the term of order $t^{1 / 2}$. This expansion is used for computing various types of curvature flows we mentioned in the Introduction.

It is convenient for calculations below to choose Cartesian coordinates $(x, y, z)$ so that the origin is situated at $\mathbf{r}_{0}$ and the $O z$-axis has the direction parallel to the normal $\mathbf{N}$. Coordinates of the boundary point $\mathbf{p}$ become $(0,0,-v t)$. Consider a cylinder $\mathcal{S}(\varepsilon)=\left\{(x, y, z) \in \mathbb{R}^{3} ; x^{2}+y^{2}<\varepsilon^{2}\right\}$ and a ball $B(\varepsilon)=\left\{(x, y, z) \in \mathbb{R}^{3} ; x^{2}+y^{2}+z^{2}<\varepsilon^{2}\right\}$. If $\partial C$ is smooth, it can be represented as a graph of a smooth function locally in some neighborhood of $\mathbf{p}$. More precisely, in our case there exists a small $t>0$ such that

$$
\partial C \cap \mathcal{S}(v t) \cap B(2 v t)=\{(x, y,-v t+f(x, y)):(x, y, 0) \in \mathcal{S}(v t)\}
$$

where $f: \mathbb{R}^{2} \rightarrow \mathbb{R}$ is a smooth function and $f(0,0)=0, \nabla f(0,0)=0$.

Theorem 1 Let $\partial C$ be smooth, $\mathbf{p} \in \partial C$ and $v \in \mathbb{R}$. Then the convolution 9 has the following asymptotic expansion as $t \rightarrow 0$ at the point $\mathbf{r}_{0}=\mathbf{p}+\mathbf{N} v t$ :

$$
\begin{aligned}
M\left(\mathbf{r}_{0}\right)= & \frac{1}{2}-t^{1 / 4} \frac{\pi m_{3}}{2} H \\
& +t^{3 / 4} \frac{\pi}{16}\left[32 m_{1} v-m_{5}\left(\Delta H+2 H\left(H^{2}-K\right)\right)\right]+O\left(t^{5 / 4}\right),
\end{aligned}
$$

where $H, K$ and $\Delta H$ are values of the mean curvature, the Gauss curvature and the LaplaceBeltrami operator of the mean curvature of $\partial C$ at the point $\mathbf{p}$ and $m_{i}=\int_{0}^{\infty} r^{i} \rho\left(r^{2}\right) \mathrm{d} r$.

Proof. We introduce the scaled variable $\tau=t^{1 / 4}$. Using the normalization, rotational symmetry, and exponential decreasing properties of $\rho\left(|\mathbf{r}|^{2}\right), M\left(\mathbf{r}_{0}\right)$ can be rewritten as follows:

$$
\begin{aligned}
M\left(\mathbf{r}_{0}\right) & =\int_{\mathbb{R}^{3}} \chi_{C}(\mathbf{r}) \rho_{t^{1 / 4}}(\mathbf{r}) \mathrm{d} \mathbf{r}=\int_{C} \rho_{t^{1 / 4}}(\mathbf{r}) \mathrm{d} \mathbf{r} \\
& =\int_{x^{2}+y^{2}<\sqrt{t}} \mathrm{~d} x \mathrm{~d} y \int_{-v t+f(x, y)}^{\sqrt{x^{2}+y^{2}}} \rho_{t^{1 / 4}}(\mathbf{r}) \mathrm{d} z+O\left(e^{-\tau^{-2}}\right) \\
& =\int_{\mathbb{R}^{2}} \mathrm{~d} x \mathrm{~d} y \int_{-v t+f(x, y)}^{+\infty} \rho_{t^{1 / 4}}(\mathbf{r}) \mathrm{d} z+O\left(e^{-\tau^{-2}}\right) \\
& =\frac{1}{2}-\int_{\mathbb{R}^{2}} \mathrm{~d} x \mathrm{~d} y \int_{0}^{-v \tau^{4}+f(x, y)} \rho_{\tau}(\mathbf{r}) \mathrm{d} z+O\left(e^{-\tau^{-2}}\right) .
\end{aligned}
$$




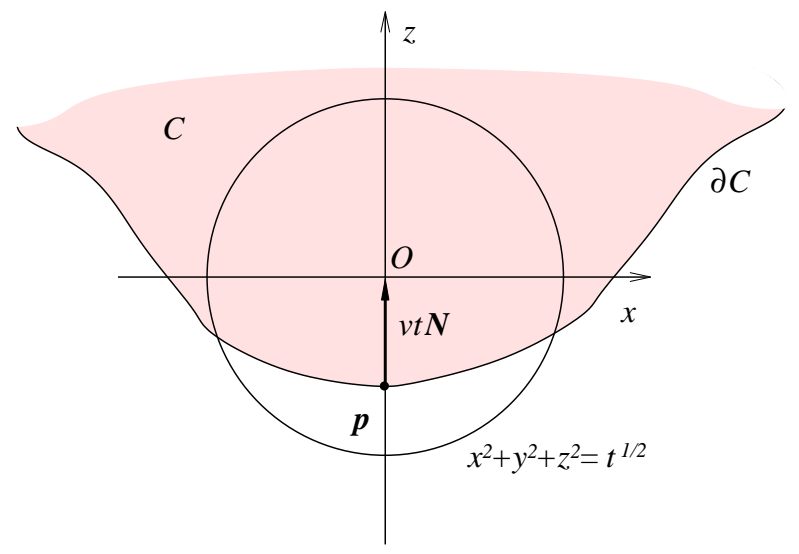

FIG. 1. Illustration for the asymptotic expansion.

We make the change of variables $\mathbf{r}^{\prime}=\mathbf{r} / \tau$ in the last integral. We also use the old notations for Cartesian coordinates for the new variable: $\mathbf{r}^{\prime}=(x, y, z)$. Therefore

$$
M\left(\mathbf{r}_{0}\right)=\frac{1}{2}-\int_{\mathbb{R}^{2}} \mathcal{I}(\tau, x, y) \mathrm{d} x \mathrm{~d} y+O\left(e^{-\tau^{-2}}\right),
$$

where

$$
\mathcal{I}(\tau, x, y)=\int_{0}^{g(\tau, x, y)} \rho\left(x^{2}+y^{2}+z^{2}\right) \mathrm{d} z \quad \text { with } \quad g(\tau, x, y)=-v \tau^{3}+\frac{f(\tau x, \tau y)}{\tau} .
$$

The integral $\int_{\mathbb{R}^{2}} \mathcal{I}(\tau, x, y) \mathrm{d} x \mathrm{~d} y$ measures the signed weighted deviation of $\partial C$ from the $(x, y)$ plane.

Observe that $\nabla f(0,0)=0$ and $f(0,0)=0$ implies that $g(\tau, x, y)$ is in fact a smooth function of $\tau$ at $\tau=0$. We expand $\mathcal{I}(\tau, x, y)$ into a Taylor series with respect to $\tau$ :

$$
\begin{aligned}
\mathcal{I}(\tau, x, y)= & \rho\left(x^{2}+y^{2}\right) \frac{\partial g}{\partial \tau}(0, x, y) \tau+\frac{1}{2} \rho\left(x^{2}+y^{2}\right) \frac{\partial^{2} g}{\partial \tau}(0, x, y) \tau^{2} \\
& +\frac{1}{6}\left(2 \rho^{\prime}\left(x^{2}+y^{2}\right)\left(\frac{\partial g}{\partial \tau}(0, x, y)\right)^{3}+\rho\left(x^{2}+y^{2}\right) \frac{\partial^{3} g}{\partial \tau^{3}}(0, x, y)\right) \tau^{3}+O\left(\tau^{4}\right) .
\end{aligned}
$$

We calculate derivatives of $g(\tau, x, y)$ with respect to $\tau$ at $\tau=0$ :

$$
\begin{aligned}
\frac{\partial g}{\partial \tau}(0, x, y)= & \frac{1}{2} y^{2} f_{22}(0,0)+x y f_{12}(0,0)+\frac{1}{2} x^{2} f_{11}(0,0), \\
\frac{\partial^{2} g}{\partial \tau^{2}}(0, x, y)= & \frac{1}{3} y^{3} f_{222}(0,0)+x y^{2} f_{122}(0,0) \\
& +x^{2} y f_{112}(0,0)+\frac{1}{3} x^{3} f_{111}(0,0),
\end{aligned}
$$




$$
\begin{aligned}
\frac{\partial^{3} g}{\partial \tau^{3}}(0, x, y)= & -6 v+\frac{1}{4} y^{4} f_{2222}(0,0)+x y^{3} f_{1222}(0,0)+\frac{3}{2} x^{2} y^{2} f_{1122}(0,0) \\
& +x^{3} y f_{1112}(0,0)+\frac{1}{4} x^{4} f_{1111}(0,0),
\end{aligned}
$$

and substitute these expressions into the expansion for $\mathcal{I}(\tau, x, y)$.

The integration of $\mathcal{I}(\tau, x, y)$ over $x$ and $y$ leads to

$$
\begin{aligned}
M\left(\mathbf{r}_{0}\right)= & \frac{1}{2}-\frac{\tau \pi m_{3}}{2}\left(f_{11}+f_{22}\right)+\frac{\tau^{3} \pi}{64}\left[128 m_{1} v\right. \\
& -m_{5}\left[\left(f_{22}+f_{11}\right)\left(5 f_{22}^{2}+12 f_{12}^{2}-2 f_{22} f_{11}+5 f_{11}^{2}\right)\right) \\
& \left.\left.-2\left(f_{2222}+2 f_{1122}+f_{1111}\right)\right]\right]+O\left(\tau^{5}\right),
\end{aligned}
$$

where all derivatives of $f$ are calculated at the origin. Recalling (3), (8) and (7) leads to the statement of the theorem:

$$
M\left(\mathbf{r}_{0}\right)=\frac{1}{2}-t^{1 / 4} \pi m_{3} H+\frac{t^{3 / 4} \pi}{16}\left[32 m_{1} v-m_{5}\left(\Delta H+2 H\left(H^{2}-K\right)\right)\right]+O\left(t^{5 / 4}\right) .
$$

In order to isolate the third term in the expansion $[10$, , consider another convolution

$$
M_{a}(\mathbf{r}, t)=\left(\chi_{C} \star \rho_{a t^{1 / 4}}\right)(\mathbf{r}),
$$

where

$$
\rho_{a t^{1 / 4}}(\mathbf{r})=\rho\left(|\mathbf{r}|^{2} /\left(a t^{1 / 4}\right)^{2}\right)\left(a t^{1 / 4}\right)^{-3} \quad \text { and } \quad a \in(0,1) .
$$

The following corollary shows that a linear combination of (12) and (9) eliminates the first two terms in the asymptotics.

Corollary 1 Let $C, p, v$ and $\mathbf{r}_{0}$ be as in Theorem 1 . Then the following asymptotic expansion holds at $\mathbf{r}_{0}$ as $t \rightarrow 0$ :

$$
\frac{a\left(\left(2 M_{a}\left(\mathbf{r}_{0}\right)-1\right)-a\left(2 M_{1}\left(\mathbf{r}_{0}\right)-1\right)\right)}{\pi\left(1-a^{2}\right)}=t^{3 / 4}\left(4 m_{1} v+a^{2} m_{5} / 8\left(\Delta H+2 H\left(H^{2}-K\right)\right)\right)+O\left(t^{5 / 4}\right) .
$$

Proof. Performing the asymptotic expansion of $M_{a}(t, \mathbf{r})$ along the lines of the previous proof we get

$$
M_{a}\left(\mathbf{r}_{0}\right)=\frac{1}{2}-a t^{1 / 4} \pi m_{3} H+\frac{t^{3 / 4} \pi}{16}\left[\frac{32 m_{1} v}{a}-a^{3} m_{5}\left(\Delta H+2 H\left(H^{2}-K\right)\right)\right]+O\left(t^{5 / 4}\right),
$$

and the statement of the corollary follows.

Given a bounded smooth domain $C$ we define the thresholding function $\Theta(t, \mathbf{r})$ at an arbitrary point $\mathbf{r} \in \mathbb{R}^{3}$ :

$$
\Theta(t, \mathbf{r})=\left(2 M_{a}(t, \mathbf{r})-1\right)-a\left(2 M_{1}(t, \mathbf{r})-1\right) .
$$

Take a small $t$ and consider the sets

$$
\begin{aligned}
C_{\text {in }}(t) & =\left\{\mathbf{r} \in C: \operatorname{dist}(\mathbf{r}, \partial C)>t^{1 / 4}\right\}, \\
C_{\text {out }}(t) & =\left\{\mathbf{r} \in \mathbb{R}^{3} \backslash C: \operatorname{dist}(\mathbf{r}, \partial C)>t^{1 / 4}\right\} .
\end{aligned}
$$


Since $\rho$ is normalized and $a \in(0,1)$, we have

$$
\Theta(t, \mathbf{r})= \begin{cases}1-a>0 & \text { if } \mathbf{r} \in C_{\text {in }}(t) \\ \Theta(t, \mathbf{r})=a-1<0 & \text { if } \mathbf{r} \in C_{\text {out }}(t)\end{cases}
$$

The next proposition states that the zero level set of $\Theta(t, \mathbf{r})$ inside the stripe $\mathbb{R}^{3} \backslash\left(C_{\text {in }}(t) \cup C_{\text {out }}(t)\right)$ approximates a single step of the Willmore evolution of $\partial C$ with time step $t$.

Proposition 1 For a smooth domain $C \subset \mathbb{R}^{3}$ define

$$
C_{1}=\left\{\mathbf{r} \in \mathbb{R}^{3}: \Theta(t, \mathbf{r}) \geqslant 0\right\}
$$

and

$$
w(\mathbf{p})=-\frac{m_{5}}{32 m_{1}}\left[\Delta H(\mathbf{p})+2 H(\mathbf{p})\left(H^{2}(\mathbf{p})-K(\mathbf{p})\right)\right]
$$

for each $\mathbf{p} \in \partial C$. Then there is $t>0$ such that

$$
\partial C_{1}=\left\{\mathbf{p}+v(\mathbf{p}) t \mathbf{N}(\mathbf{p}): \mathbf{p} \in \partial C, v(\mathbf{p})=a^{2} w(\mathbf{p})+O\left(t^{1 / 2}\right)\right\} .
$$

Proof. By construction (see Corollary 1 , one zero level set of $\Theta$ lies $O(t)$ close to $\partial C$, therefore

$$
\partial C_{1} \supseteq\left\{\mathbf{p}+v(\mathbf{p}) t \mathbf{N}(\mathbf{p}): \mathbf{p} \in \partial C, v(\mathbf{p})=a^{2} w(\mathbf{p})+O\left(t^{1 / 2}\right)\right\} .
$$

Furthermore, far from $\partial C$, namely on $C_{\text {in }}$ and $C_{\text {out }}, \Theta$ is different from zero. We just need to show that it has no additional zero level sets in the stripe

$$
\left\{\mathbf{r} \in \mathbb{R}^{3}: \operatorname{dist}(\mathbf{r}, \partial C)=O\left(t^{\xi}\right), 1 / 4<\xi<1\right\} .
$$

The asymptotics of $M_{a}$ in this region is obtained similarly to the expansion of (12). It reads

$$
M_{a}\left(t, \mathbf{p}+v t^{\xi} \mathbf{N}\right)=M_{a}(t, \mathbf{p})+4 \pi m_{1} v a^{-1} t^{\xi-1 / 4}+O\left(t^{2 \xi-1 / 2}\right),
$$

where

$$
M_{a}(t, \mathbf{p})=\frac{1}{2}-a t^{1 / 4} \pi m_{3} H(\mathbf{p})+2 \pi t^{3 / 4} a^{3} m_{1} w(\mathbf{p})+O\left(t^{5 / 4}\right)
$$

Therefore

$$
\Theta\left(t, \mathbf{p}+v t^{\xi} \mathbf{N}\right)=4 \pi m_{1} v\left(\frac{1-a^{2}}{a}\right) t^{\xi-1 / 4}+O\left(t^{3 / 4}\right)+O\left(t^{2(\xi-1 / 4)}\right) .
$$

Thus, if $\Theta$ is computed inside $C$, then $v>0$ and the lowest order term in the above expansion would be greater than zero. This leads to positivity of $\Theta$ in that region. In the case when $v<0, \Theta$ is negative for small enough $t$.

In other words, we have established that for small $t>0$,

$$
\partial(\{\mathbf{r}: \Theta(t, \mathbf{r}) \geqslant 0\})=\{\mathbf{p}+v(\mathbf{p}) t \mathbf{N}(\mathbf{p}): \mathbf{p} \in \partial C\},
$$

where

$$
v(\mathbf{p})=-\frac{a^{2} m_{5}}{32 m_{1}}\left(\Delta H(\mathbf{p})+2 H(\mathbf{p})\left(H^{2}(\mathbf{p})-K(\mathbf{p})\right)\right)+O\left(t^{1 / 2}\right) .
$$

These results motivate introducing an operator $T(t)$ acting on the family $\mathbb{K}$ of domains $C$ with smooth boundaries by

$$
T(t) C=\left\{\mathbf{r} \in \mathbb{R}^{3}: \Theta(t, \mathbf{r}) \geqslant 0\right\} .
$$


Proposition 2 Let $C \in \mathbb{K}$. The boundary $\partial C_{1}$ of $C_{1}=T(t) C$ is smooth for small enough values of $t$.

Proof. Since $\partial C_{1}$ is the zero level set of the thresholding function $\Theta(t, \mathbf{r})$, it is enough to show that $\nabla \Theta(t, \mathbf{r}) \neq 0$ on it. To see this, we take small $t$ and expand the value of each component of $\nabla M_{a}$ into the power series in $t$. Since we are interested in the values of $\nabla M_{a}$ on $\partial C_{1}$ we use the same system of coordinates and the scaled variable $\tau=t^{1 / 4}$ as in the proof of Theorem 1 . We obtain

$$
\begin{aligned}
\nabla M_{a}\left(\mathbf{r}_{0}\right) & =\int_{\mathbb{R}^{3}} \chi_{C}(\mathbf{r}) \nabla \rho_{a t^{1 / 4}}(\mathbf{r}) \mathrm{d} \mathbf{r}=\int_{C} \frac{2 \mathbf{r} \rho^{\prime}\left(|\mathbf{r}|^{2} /\left(a^{2} t^{1 / 2}\right)\right)}{\left(a t^{1 / 4}\right)^{5}} \mathrm{~d} \mathbf{r} \\
& =\int_{\mathbb{R}^{2}} \int_{0}^{\infty} \frac{2 \mathbf{r} \rho^{\prime}\left(|\mathbf{r}|^{2} /\left(a^{2} t^{1 / 2}\right)\right)}{\left(a t^{1 / 4}\right)^{5}} \mathrm{~d} z \mathrm{~d} x \mathrm{~d} y-\int_{\mathbb{R}^{2}} \mathcal{J}(\tau, x, y) \mathrm{d} x \mathrm{~d} y+O\left(e^{-\tau^{-2}}\right) \\
& =\left(0,0,-\frac{2 \pi m_{1}}{a t^{1 / 4}}\right)-\int_{\mathbb{R}^{2}} \mathcal{J}(\tau, x, y) \mathrm{d} x \mathrm{~d} y+O\left(e^{-\tau^{-2}}\right)
\end{aligned}
$$

where

$$
\begin{aligned}
\mathcal{J}(\tau, x, y) & =\frac{1}{a \tau} \int_{0}^{g(\tau, x, y)}\left(0,0, \frac{\partial}{\partial z} \rho\left(x^{2}+y^{2}+z^{2}\right)\right) \mathrm{d} z \\
& =\frac{1}{a \tau}\left(0,0,-\rho\left(x^{2}+y^{2}\right)+\rho\left(x^{2}+y^{2}+g^{2}(\tau, x, y)\right)\right)
\end{aligned}
$$

with

Since $g(0, x, y)=0$, we have

$$
g(\tau, x, y)=-v \tau^{3}+\frac{f(\tau x, \tau y)}{\tau} .
$$

$$
\nabla M_{a}\left(\mathbf{r}_{0}\right)=\left(0,0,-\frac{2 \pi m_{1}}{a t^{1 / 4}}+O\left(t^{1 / 4}\right)\right), \quad t \rightarrow 0,
$$

and

$$
\begin{aligned}
\nabla \Theta\left(t, \mathbf{r}_{0}\right) & =2\left(\nabla M_{a}\left(t, \mathbf{r}_{0}\right)-a \nabla M_{1}\left(t, \mathbf{r}_{0}\right)\right) \\
& =4 \pi\left(0,0, \frac{\left(a^{2}-1\right) m_{1}}{a t^{1 / 4}}+O\left(t^{1 / 4}\right)\right) \neq 0, \quad t \rightarrow 0 .
\end{aligned}
$$

The above proposition shows that for $t$ small enough $T(t)$ acts from $\mathbb{K}$ to $\mathbb{K}$. Therefore we can define for the same $t$ the following operator $\Gamma(t)$ acting on smooth embeddings of $\Sigma$. For the embedding $f: \Sigma \rightarrow \mathbb{R}^{3}$ with $f(\Sigma)=\partial C$ we define $\Gamma(t) f=f_{1}$ by the shift $v t$ along the normal $\mathbf{N}$ to $\partial C$ :

$$
f_{1}(\sigma)=f(\sigma)+t v \mathbf{N}
$$

such that $\Theta\left(f_{1}(\sigma)\right)=0$ and therefore $f_{1}(\Sigma)=\partial C_{1}$.

Next we consider some finite $t$. Taking a large $m \in \mathbb{N}$ we have

$$
T^{m}\left(\frac{t}{m}\right): \mathbb{K} \rightarrow \mathbb{K}
$$

In the next theorem we state the convergence

$$
\partial\left(T^{m}\left(\frac{t}{m}\right) C\right) \rightarrow f(t, \Sigma) \quad \text { as } m \rightarrow \infty
$$


and

$$
\Gamma^{m}\left(\frac{t}{m}\right) f_{0} \rightarrow f(t, \cdot) \quad \text { as } m \rightarrow \infty,
$$

where $f(t, \sigma)$ denotes the Willmore flow of $\Sigma$ with initial data $f_{0}$.

THEOREM 2 Suppose $f_{0}: \Sigma \rightarrow \mathbb{R}^{3}$ is an embedding of a smooth closed two-dimensional manifold $\Sigma$ such that $f_{0}(\Sigma)=\partial C_{0}$ is the boundary of a bounded domain $C_{0} \subset \mathbb{R}^{3}$. Assume the existence of a smooth embedded Willmore flow $f(t, \sigma)$ on the time interval $[0, T]$ with initial data $f_{0}$. We introduce a scaling for the time variable $t$ such that the coefficient in the main term in 15) is equal to 1 . For $k, m$ integer, $t \in(0, T], \sigma \in \Sigma$ we denote by $\hat{f}_{k}(t / m t, \sigma)$ the approximate solution, namely an embedding of $\Sigma$ in $\mathbb{R}^{3}$ such that $\hat{f}_{k}(t / m, \sigma)=\Gamma(t / m)\left(\hat{f}_{k-1}(t / m, \sigma)\right)$ and $\hat{f}_{0}(t / m, \sigma)=f_{0}(\sigma)$. Then $\hat{f}_{m}(t / m, \sigma) \rightarrow f(t, \sigma)$ as $m \rightarrow \infty$ locally in $t \in[0, T]$ uniformly in $\sigma \in \Sigma$.

Proof. We omit the argument $\sigma$ in $f(t, \sigma)$ and introduce the following notations. $\mathbf{N}(t)$ is the normal vector of $f(t), w(t)$ is the normal velocity of the Willmore flow $f(t)$ :

$$
w(t)=-\Delta H(t)-2 H(t)\left(H^{2}(t)-K(t)\right),
$$

and $f_{k}=f(k t / m), w_{k}=w(k t / m), \mathbf{N}_{k}=\mathbf{N}(k t / m)$. Similar variables corresponding to the approximate solution $\hat{f}_{k}(t / m, \sigma)$ are denoted by $\hat{f}_{k}, \hat{w}_{k}, \hat{\mathbf{N}}_{k}$. The shift in the normal direction between consecutive positions of the approximate solution is $(t / m) \hat{v}_{k}$, namely $\hat{f}_{k+1}=\hat{f}_{k}+$ $\hat{v}_{k}(t / m) \hat{\mathbf{N}}_{k}$. In this sense $\hat{v}_{k}$ is the velocity of the approximate solution $\hat{f}_{k}$.

Then for $t \in(0, T]$,

$$
f_{1}=f_{0}+\int_{0}^{t / m} \mathbf{N}(t) w(t) \mathrm{d} t=f_{0}+\frac{t}{m} \mathbf{N}_{0} w_{0}+O\left((t / m)^{2}\right) .
$$

The equality (16) follows from the assumption that the solution $f(t)$ is smooth. According to [15,

$$
w_{0}=\hat{v}_{0}+O\left((t / m)^{1 / 2}\right) .
$$

Therefore

$$
f_{1}=\hat{f}_{1}+O\left((t / m)^{3 / 2}\right) \text {. }
$$

This implies

$$
\mathbf{N}_{1}=\hat{\mathbf{N}}_{1}+O\left((t / m)^{3 / 2}\right)
$$

and

$$
w_{1}=\hat{w}_{1}+O\left((t / m)^{3 / 2}\right)=\hat{v}_{1}+O\left((t / m)^{1 / 2}\right),
$$

where the last equality is again due to $\left[15\right.$. Now we can repeat the argument for $f_{1}$ considering $f_{2}$ to get

$$
f_{2}=f_{1}+\int_{t / m}^{2 t / m} N(t) w(t) d t=f_{1}+\frac{t}{m} N_{1} w_{1}+O\left((t / m)^{2}\right)=\hat{f}_{2}+O\left((t / m)^{3 / 2}\right) .
$$

This leads to

$$
f_{m}-\hat{f}_{m}=\frac{t}{m} \sum_{i=0}^{m-1}\left(N_{i} w_{i}-\hat{N}_{i} \hat{w}_{i}\right)+O\left(t^{2} / m\right)=O\left((1 / m)^{1 / 2}\right),
$$

and the convergence follows. 


\section{Numerical implementation and examples}

In this section we briefly describe the implementation of the convolution thresholding algorithm developed above. We also present several computed examples of Willmore evolution. To see how the method performs when the initial surface is not smooth, we use homothetic Willmore evolutions found in [10].

\subsection{Implementation using the Fourier series}

Given a bounded domain $C \subset(0,1)^{3}$, to approximate the Willmore evolution of $\partial C$ on $t \in$ $\left[0, t_{e}\right]$, we construct domains $C_{i}$ and corresponding surfaces $\partial C_{i}$ for time instants $t_{i}=i \Delta t$, $i=0,1, \ldots, n_{e}\left(n_{e}=\left\lfloor t_{e} / \Delta t\right\rfloor\right)$, by the following algorithm:

1. Set $C_{0}=C$.

2. Repeat for $i=0, \ldots, n_{e}-1$ :

(a) Convolution, i.e. construction of

$$
M_{a_{k}}(\mathbf{r})=\left(\chi_{C_{i}} \star \rho_{a_{k} \Delta t^{1 / 4}}\right)(\mathbf{r})
$$

where $k=1,2$ with $a_{1}=1$ and $a_{2} \in(0,1)$.

(b) Computing the thresholding function $\Theta(t, \mathbf{r})$,

$$
\Theta(\Delta t, \mathbf{r})=\left(2 M_{a_{2}}(\Delta t, \mathbf{r})-1\right)-a_{2}\left(2 M_{a_{1}}(\Delta t, \mathbf{r})-1\right) .
$$

(c) Thresholding, i.e. localization of the next position of the surface as the boundary of

$$
C_{i+1}=\left\{\mathbf{r} \in \mathbb{R}^{3}: \Theta(\Delta t, \mathbf{r}) \geqslant 0\right\} .
$$

First, consider the thresholding (2c) including the approximation of the zero level set of the thresholding function $\Theta$. This step of the procedure can be performed by means of a modified Marching Cubes algorithm [17]. It was adapted and applied to the mean curvature flow calculations in [22] and [23].

Given an initial cubic grid $\left\{0, h_{0}, 2 h_{0}, \ldots, 1\right\}^{3}$ and a domain $C_{i}$ we compute the values of $\Theta$ at all grid nodes. Next, the cubes where the computed values have different signs are identified. Since the zero level set passes through these cubes, each of them is divided into eight similar ones. Then $\Theta$ is evaluated at the new nodes. This adaptive refinement procedure is repeated $L$ times, so that the desired space resolution $h_{0} 2^{-L}$ is reached. The cubes of the smallest size are the only ones that are crossed by the zero level set. Each such cube is divided into six tetrahedrons and the function $\Theta$ is interpolated linearly inside each tetrahedron using the already computed values at the vertices. The zero level set inside each tetrahedron is approximated by one or two triangles. The union of these triangles gives the approximation to the zero level set of $\Theta$. At the same time an adaptive spatial discretization of the set $C_{i+1}$ that is the inside of $\partial C_{i+1}$ is created:

$$
C_{i+1}=\left(\bigcup_{j} \mathbf{K}_{j} \cup \bigcup_{k} \mathbf{T}_{k}\right) \cup R .
$$

Here $\mathbf{K}_{j}$ are cubes, $\mathbf{T}_{k}$ are tetrahedrons, the unions are disjoint up to a set of measure zero and the volume of the remaining set $R$ satisfies

$$
\text { Vol } R \leqslant O\left(2^{-2 L}\right) .
$$


To compute the convolutions $M_{a_{k}}$ we extend $\chi_{C_{i}}$ periodically to the whole $\mathbb{R}^{3}$ and use Fourier series. Numerical aspects of similar computations in the case of the mean curvature evolution have been presented in [22, 23] and [11].

To compute the Fourier coefficients $c_{j k l}$ of $\chi_{C_{i}}$ the following integrals have to be evaluated for $j, k, l=0, \ldots, N$ :

$$
c_{j k l}=\int_{C_{i}} \exp (2 \pi \mathbf{i}(j x+k y+l z)) \mathrm{d} x \mathrm{~d} y \mathrm{~d} z .
$$

After substituting the decomposition (18), the integration of the exponent over the cubes $\mathbf{K}_{j}$ and tetrahedrons $\mathbf{T}_{k}$ has to be performed. The integrals over $\mathbf{K}_{j}$ are expressed as a weighted sum of eight exponents. Furthermore, since the size of each tetrahedron is small (diam $\mathbf{T}_{k}<h_{0} 2^{-L}$ ), the integrals over $\mathbf{T}_{k}$ are well approximated by Gauss quadratures of order $m_{g}$. Thus, the computation of the Fourier coefficients is reduced to the evaluations of the sums

$$
c_{j k l}=\sum_{m=1}^{N_{p}} g_{m} \exp \left(2 \pi \mathbf{i}\left(j x_{m}+k y_{m}+l z_{m}\right)\right)+O\left(2^{-m_{g} L}\right)+O\left(2^{-2 L}\right),
$$

where the points $\left\{x_{m}, y_{m}, z_{m}\right\}_{m=1}^{N_{p}}$ are non-uniformly spaced, and $g_{m}$ are some weights. It is clear from (19) that $m_{g}=2$ is the optimal choice. This means that it is enough to take just one Gauss point inside each tetrahedron.

A fast algorithm to approximately evaluate sums (19) can be found in [2]. The numerical cost of evaluating all $N^{3}$ coefficients by means of this algorithm is

$$
O\left(\beta^{3} N_{p}+N^{3} \log (N)\right),
$$

where $\beta$ is a constant depending on the desired accuracy. In the case $\beta=23$ this accuracy is comparable with the machine truncation error.

With $c_{j k l}$ at hand, the Fourier coefficients $b_{j k l}^{(i)}$ of the convolutions $M_{a_{i}}$ are obtained from

$$
b_{j k l}^{(i)}=c_{j k l} r_{j k l}^{(i)},
$$

where $r_{j k l}^{(i)}$ are the precomputed Fourier coefficients of the convolution kernel $\rho_{a_{i} \Delta t^{1 / 4}}$.

The evaluation of the thresholding function $\Theta$ at an arbitrary point involves backward summation

$$
\Theta(\Delta t, \mathbf{r})=a_{2}-1+2 \sum_{j, k, l=0}^{N}\left(b_{j k l}^{(2)}-a_{2} b_{j k l}^{(1)}\right) \exp (-2 \pi \mathbf{i}(j, k, l) \cdot \mathbf{r}) .
$$

To avoid a cost of $N^{3}$ operations for each evaluation, an accurate interpolation procedure described in [2] is used. We first evaluate the sums on the uniform grid by computing the fast Fourier transform. Then the value of $\Theta$ at an arbitrary point is interpolated by means of central B-splines of order $\beta$. The cost of one such interpolation is $O\left(\beta^{3}\right)$ operations.

\subsection{Computed examples}

The results presented here were obtained using the exponential convolution kernel

$$
\begin{aligned}
\rho(x) & =\pi^{-3 / 2} \exp (-x), \\
\rho_{\Delta t^{1 / 4}}(\mathbf{r}) & =(\pi \sqrt{\Delta t})^{-3 / 2} \exp \left(-\frac{\mathbf{r} \cdot \mathbf{r}}{\sqrt{\Delta t}}\right) .
\end{aligned}
$$


The main parameter of the computational procedure is the time step $\Delta t$. It is adapted to the geometry of the evolving surface. Values of other parameters, i.e. the maximal level of grid refinement $L$ and the number of the Fourier modes follow from the balance of approximation errors. Consider a smooth initial surface depicted in Figure 2 The value of $\Delta t$ has to scale the essential support of the convolution kernel so that all geometrical features of the surface are resolved. The kernel (21) has more than $99.95 \%$ of its mass inside the ball of radius $3 \Delta t^{1 / 4}$. Since the minimal curvature radius of the initial surface is about 0.05 , the time step has to be chosen to be $\Delta t=10^{-8}$. This way the surface inside the support of the convolution kernel can be represented as a graph, and the asymptotic expansion in Theorem 1 is valid. In these settings we choose the size of the smallest grid cell to be 1/256, and the exponential decay of the Fourier coefficients of the kernel suggests taking $N=128$. A nearly optimal complexity of the computational procedure makes it possible to run the simulation of this example on a rather modest PC. On a SPARC $333 \mathrm{Mhz}$ CPU with $512 \mathrm{MB}$ RAM the computation of one time step took about 20 seconds.
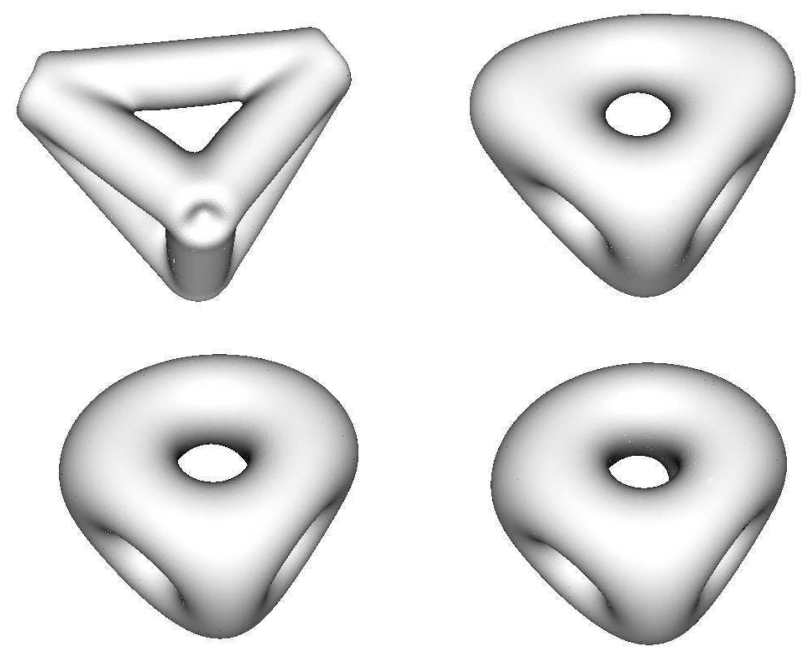

FIG. 2. Evolution of a non-convex surface: time step is $10^{-8}$, step numbers $0,80,480$ and 1080 .

We now turn to non-smooth initial data. As a test example we consider a Willmore flow originating from a sharp cone described in [10]. The cone evolves self-similarly as $f(t, \mathbf{x})=$ $f_{*}(\mathbf{x}) \cdot C_{s} t^{1 / 4}$, where $f_{*}(\mathbf{x})$ is a time independent embedding, and $C_{s}$ is a shape dependent constant. We take a sharp cone with angle $\alpha$ as an initial surface and compute the Willmore evolution by the method of the present paper. In Figure 3 we compare the cross-section of the evolving surface taken after 10 time steps with the self-similar solution obtained by solving numerically a system of ODEs for $f_{*}(\mathbf{x})$ (see [10]). We observe a good agreement of the results. Furthermore, the selfsimilar Willmore evolution implies that the coordinate of the vertex of the cone $r_{v}(t)$ changes in time according to the following simple law:

$$
r_{v}(t)=C_{\alpha} t^{1 / 4}
$$

where $C_{\alpha}$ is a constant depending only on the angle $\alpha$. We point out that the velocity at time $t=0$ is infinite. In Figure 4 we present the comparison of the vertex coordinate position by this law with 


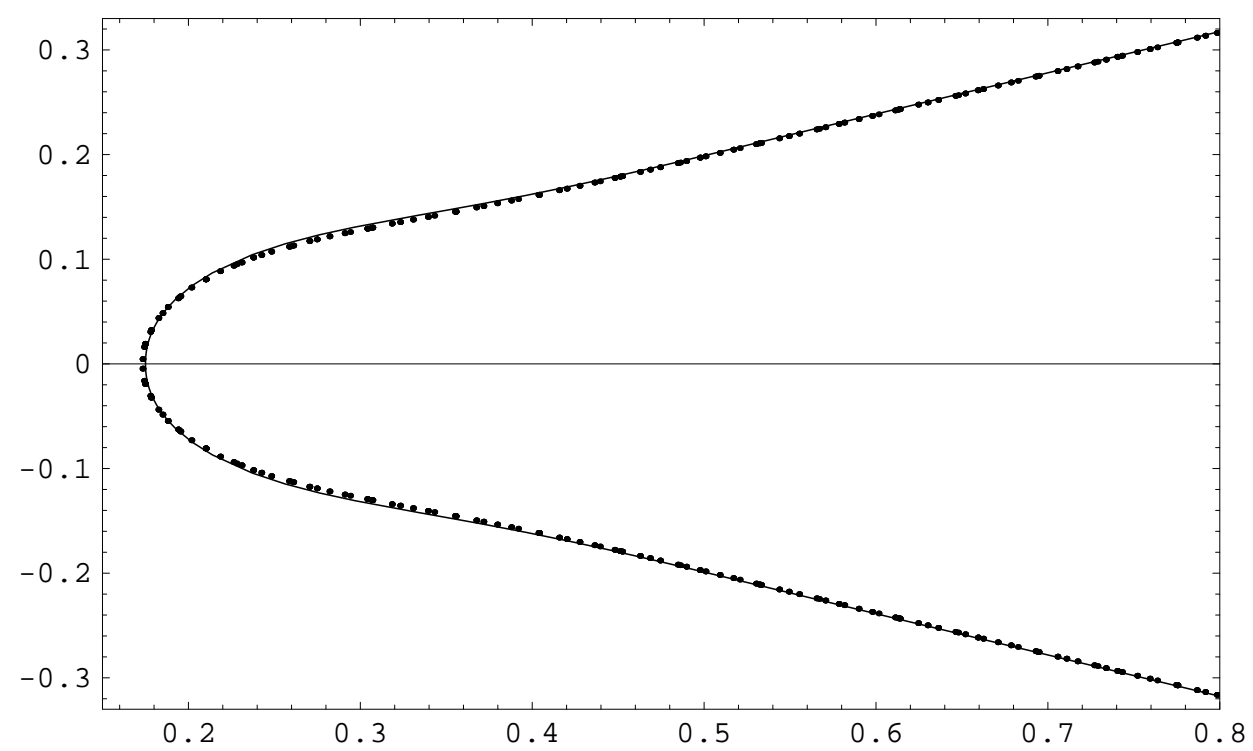

FIG. 3. Calculated cross-section of the cone after a time interval $T=1.6 \cdot 10^{-6}$ of the Willmore evolution (dots). Time step is $1.6 \cdot 10^{-7}$. The continuous line is the corresponding solution of the system of ODEs found in [10].

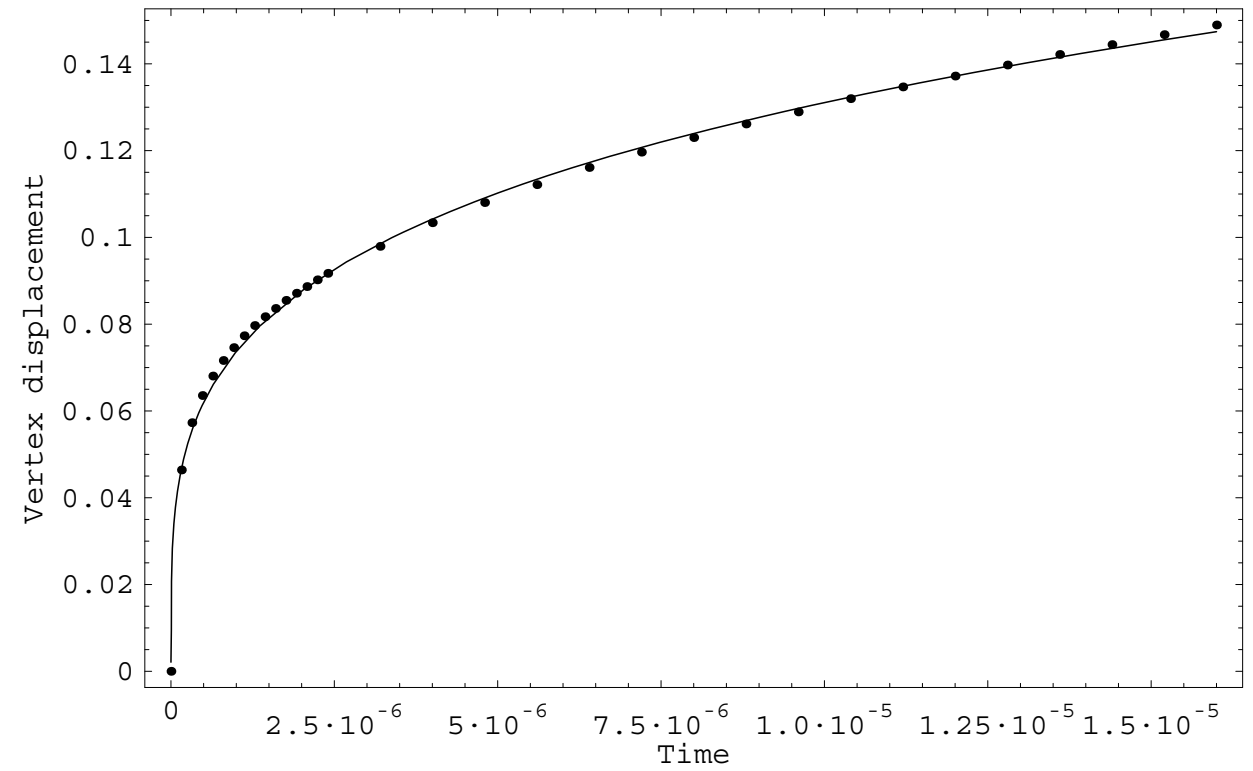

FIG. 4. The coordinate of the vertex of the cone: time step is $1.6 \cdot 10^{-7}$, step numbers $0, \ldots, 15$ and $5 i, i=4,5,6, \ldots 20$. The continuous line is the graph of the function $2.33 x^{1 / 4}$. 

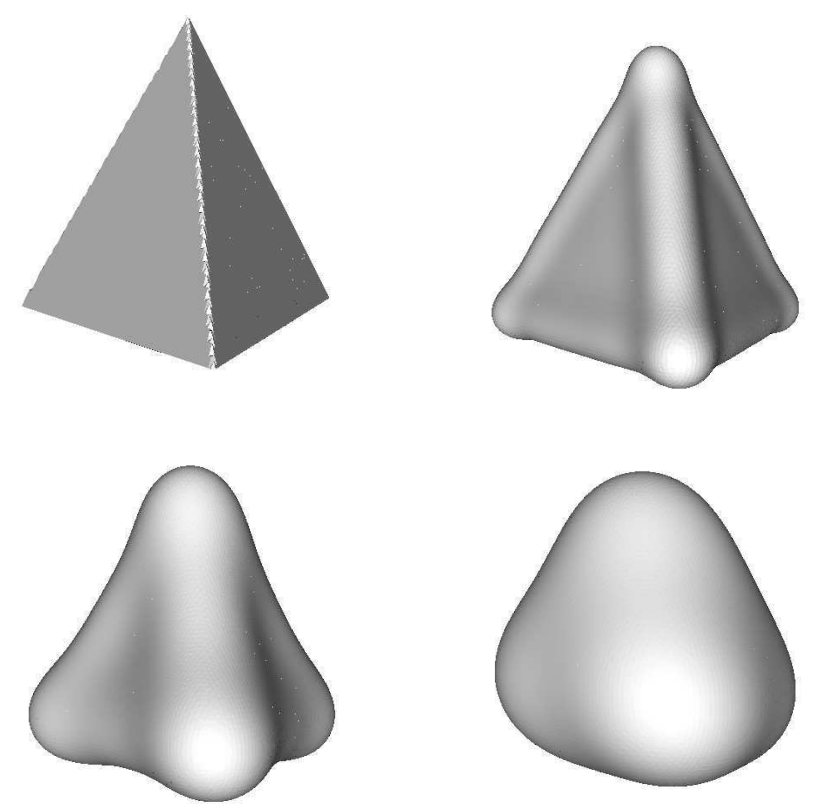

FIG. 5. The evolution of a pyramid: time step is $10^{-8}$, step numbers $0,10,110$ and 610 .

computation by our convolution thresholding method. The good agreement of results in this case indicates that the violation of the smoothness requirements for convolution thresholding at the initial time step does not spoil the approximation in the long run.

Another example of Willmore evolution of an initially non-smooth surface is depicted in Figure 5 .

\section{REFERENCES}

1. Bence, J., Merriman, B., \& Osher, S. Diffusion generated motion by mean curvature. Computational Crystal Growers Workshop, 73 (1992).

2. Beylkin, G. On the fast Fourier transform of functions with singularities. Appl. Comput. Harmon. Anal. 2 (1995), 363-381. Zbl 0838.65142 MR 1354915

3. CAnham, P. B. The minimum energy of bending as a possible explanation of the biconcave shape of the human red blood cell. J. Theor. Biol. 26 (1970), 61-81.

4. Chopp, D. L., \& Sethian, J. A. Motion by intrinsic Laplacian of curvature. Interfaces Free Bound. 1 (1999), 107-123. Zbl 0938.65144 MR 1865108

5. Clarenz, U., Diewald, U., Dziuk, G., Rumpf, M., \& Rusu, R. A finite element method for surface restoration with smooth boundary conditions. Comput. Aided Geom. Design 21 (2004), 427-445. Zbl 1069.65546 MR 2058390

6. Deuling, H. J., \& HelFrich, W. Red blood cell shapes as explained on the basis of curvature elasticity. Biophys. J. 16 (1976), 861-868.

7. Droske, M., \& RumpF, M. A level set formulation for Willmore flow. Interfaces Free Bound. 6 (2004), 361-378. Zbl 1062.35028 MR 2095338 
8. Du, Q., LiU, C., RYhaM, R., \& WANG, X. Modeling the spontaneous curvature effects in static cell membrane deformations by a phase field formulation. Comm. Pure Appl. Anal. 4 (2005), 537-548. Zbl 1078.92004 MR 2167186

9. Du, Q., LiU, C., RyHAM, R., \& WANG, X. A phase field formulation of the Willmore problem. Nonlinearity 18 (2005), 1249-1267. Zbl 1125.35366 MR 2134893

10. GrZhibovskis, R. Willmore flow and self-similar evolution of surfaces. Chalmers Univ. of Tech., Preprint 29 (2004).

11. GRZhibovskis, R., \& HeINTZ, A. A convolution-thresholding approximation of generalized curvature flows. SIAM J. Numer. Anal. 42 (2005), 2652-2670. Zbl 1084.65100 MR 2139409

12. IsHII, H. A generalization of the Bence, Merriman and Osher algorithm for motion by mean curvature. Curvature Flows and Related Topics (Levico, 1994), Gakkōtosho, Tokyo (1995), 111-127. Zbl 0844.35043 MR 1365304

13. Ishit, H., Pires, G. E., \& Souganidis, P. E. Threshold dynamics type approximation schemes for propagating fronts. J. Math. Soc. Japan 51 (1999), 267-308. Zbl 0935.53006 MR 1674750

14. Kuwert, E., \& Schätzle, R. The Willmore flow with small initial energy. J. Differential Geom. 57 (2001), 409-441. Zbl 1035.53092 MR 1882663

15. Kuwert, E., \& Schätzle, R. Gradient flow for the Willmore functional. Comm. Anal. Geom. 10 (2002), 307-339. Zbl 1029.53082 MR 1900754

16. KUWERT, E., \& SCHÄtZLE, R. Removability of point singularities of Willmore surfaces. Ann. of Math. (2) 160 (2004), 315-357. Zbl 1078.53007 MR 2119722

17. Lorensen, W., \& Cline, H. Marching cubes: A high resolution 3d surface construction algorithm. ACM Computer Graphics 21 (1987), 163-170.

18. Mayer, U. F., \& Simonett, G. A numerical scheme for axisymmetric solutions of curvature-driven free boundary problems, with applications to the Willmore flow. Interfaces Free Bound. 4 (2002), 89-109. Zbl 1005.65095 MR 1877537

19. Mayer, U. F., \& Simonett, G. Self-intersections for Willmore flow. Evolution Equations: Applications to Physics, Industry, Life Sciences and Economics (Levico Terme, 2000), Progr. Nonlinear Differential Equations Appl. 55, Birkhäuser, Basel (2003), 341-348. Zbl 1046.53046 MR 2013200

20. Merriman, B., Bence, J. K., \& Osher, S. J. Motion of multiple functions: a level set approach. J. Comput. Phys. 112 (1994), 334-363. Zbl 0805.65090 MR 1277282

21. Moser, R. Weak solutions of the Willmore flow. Max Planck Institute for Mathematics in the Sciences, Preprint No. 97 (2001).

22. RUUTh, S. Efficient algorithms for diffusion-generated motion by mean curvature. Ph.D. thesis, Univ. of British Columbia (1996).

23. Ruuth, S. J., \& Merriman, B. Convolution-thresholding methods for interface motion. J. Comput. Phys. 169 (2001), 678-707. Zbl 0988.65094 MR 1836529

24. Simonett, G. The Willmore flow near spheres. Differential Integral Equations 14 (2001), 1005-1014. Zbl pre01832854 MR 1827100

25. TAsdizen, T., Whitaker, R., Burchard, P., \& Osher, S. Geometric surface smoothing via anisotropic diffusion of normals. Proceedings of the Conference on Visualization 1 (2002), 125-132.

26. Willmore, T. J. Riemannian Geometry. Clarendon Press, Oxford (1993). Zbl 0797.53002 MR 1261641 